Check for updates

Cite this: RSC Adv., 2019, 9, 25075

Received 8th May 2019

Accepted 28th July 2019

DOI: $10.1039 / c 9 r a 03472 b$

rsc.li/rsc-advances

\title{
Amino-BODIPY as the ratiometric fluorescent sensor for monitoring drug release or "power supply" selector for molecular electronics $\uparrow$
}

\author{
Martin Porubský, ${ }^{a}$ Soňa Gurská, ${ }^{b}$ Jarmila Stanková, ${ }^{\mathrm{b}}$ Marián Hajdúch, ${ }^{\mathrm{b}}$ Petr Džubák ${ }^{\mathrm{b}}$ \\ and Jan Hlaváč iD *a
}

\begin{abstract}
The glutathione cleavable conjugates of amino-BODIPY dye with model drugs have been tested for monitoring the drug release via ratiometric fluorescence based on two excitation and one emission wavelength. As a self-immolative linker was used for the construction of conjugates, free amino-BODIPY was released with the drug. Different excitation profiles of the dye before and after conjugate cleavage and similar emission wavelengths that enabled monitoring the release of the drug via the OFF-ON effect were successfully tested inside the cancer cells. UV/Vis spectrometry could be used in the quantification of the conjugate/drug in an analyte irrespective of the cleavage grade. As the system functionality was based only on the altered acylamino-BODIPY present in the conjugate to amino-BODIPY released during the cleavage, the method could be applied as a ratiometric fluorescence theranostic system to other non-fluorescent drugs. Moreover, the present conjugates demonstrated their potential application in molecular electronics as a "power supply" selector enabling the application of two power sources for one "bulb" to maintain its light intensity.
\end{abstract}

\section{Introduction}

The ability to monitor a drug's fate, including its conjugate penetration and the subsequent drug release is one of the key features in the development of new drug delivery systems (DDS) in cancer therapy. ${ }^{1}$ The detection of relevant markers along with monitoring the drug release with time ranks these systems among the most intensively studied theranostics. ${ }^{2}$

The most frequently used visualization method for drug release is optical fluorescent spectroscopy, using various dyes such as cyanines, xanthene dyes, coumarines, etc. The frequently-used BODIPY dyes with total neutral charge, hydrophobic nature, and adjustable photochemical properties seem to be the first candidates of choice for penetration-visualization studies. In addition, BODIPYs are highly photostable and possess high quantum yields, high extinction coefficients, and sharp excitation/emission spectra. ${ }^{3}$

Optical imaging often utilizes activatable probes that effectuate amplified signal in the presence of selective or overexpressed biomarker or due to specific molecular events. This

\footnotetext{
${ }^{a}$ Department of Organic Chemistry, Faculty of Science, Palacký University, Tr. 17. Listopadu 12, 77146 Olomouc, Czech Republic. E-mail: jan.hlavac@upol.cz

${ }^{b}$ Institute of Molecular and Translational Medicine, Faculty of Medicine and Dentistry, Palacký University, Hnèvotínská 5, 779 00, Olomouc, Czech Republic

$\dagger$ Electronic supplementary information (ESI) available: Description of synthesis protocols, spectral properties of the probes and fluorescence spectroscopy recording of probes cleavage. See DOI: 10.1039/c9ra03472b
}

phenomenon is fundamental for the OFF-ON or ON-OFF systems used in several diagnostic applications. ${ }^{4-10}$ The greatest advantage of the fluorescent systems applied in chemical biology is the ratiometric measurement, ${ }^{\mathbf{1 1 - 1 3}}$ when two emission maxima are reached after excitation at one wavelength or when two excitation wavelengths cause one emission peak. Ratiometric systems easily overcome some drawbacks of simple intensiometric systems, especially false response caused by variation in the local concentration of the probe, light scattering by the sample matrix, excitation source fluctuation, or microenvironment effects around the probe.

Thiols, mainly glutathione (GSH), responsible for different redox state of the cancer cells, ${ }^{\mathbf{1 4}}$ are the key intracellular stimuli for the release of a drug from DDS or theranostics. Consequently, the level of glutathione in some tumors is up to 10-fold higher as compared to normal cells. ${ }^{15}$ Elevated GSH level is typical of some cancer cells, ${ }^{16}$ which makes them ideal triggers for the release of a drug from systems including disulfide linker.

Although a few disulfide theranostic prodrugs with an ability to monitor drug releasing via fluorescence have been introduced in recent years, ${ }^{17-22}$ only a few were reported to have the ratiometric ability for monitoring the drug release. ${ }^{23,24}$ These ratiometric OFF-ON systems are responsive to the presence of GSH and are based on one excitation/two emissions. However, the drawback of these systems is the application of the fluorescence resonance energy transfer (FRET) between camptothecin as the drug and the fluorescent dye. Thus, this system 
lacks the general application to drugs without regards to their fluorescent properties.

In the molecular electronics, the $\mathrm{OFF}-\mathrm{ON}$ or $\mathrm{ON}-\mathrm{OFF}$ concept could be used for the construction of molecular logic gates operated by various inputs, such as $\mathrm{pH}$, the presence of metal ions, or other specific markers. ${ }^{25}$ Although the molecular switches based on the (ir)reversible turn-on or turn-off effect giving the fluorescence response in the presence of appropriate marker has been described several times, ${ }^{\mathbf{2 6}}$ a selector as a molecular electronic device that can switch two different power supplies for one appliance while maintaining the level of power has not yet been described.

In this study, we report a new acylamino-BODIPY/aminoBODIPY system applicable in chemical biology or molecular electronics. As the specific spectral properties of the aminoBODIPY are exhibited together with the drug after conjugate cleavage, the drug release can be monitored via OFF-ON effect as well as ratiometric fluorescence irrespective of the fluorescent properties of the drug. The system could also serve as a molecular electronic selector activated by the presence of thiols and optimal pH.

\section{Experimental section}

\section{Materials and methods}

For the preparation and characterization of the compounds, LC/ MS analyses were performed using UHPLC/MS with an UHPLC chromatograph (Acquity) with a PDA detector, a single quadrupole mass spectrometer (Waters), an X-Select C18 column at $30{ }^{\circ} \mathrm{C}$ and a flow rate of $600 \mu \mathrm{min}^{-1}$. The mobile phase consisted of (A) $0.01 \mathrm{M}$ ammonium acetate in water and (B) acetonitrile, with a linear gradient over the course of $2.5 \mathrm{~min}$; at the conclusion of the gradient, the final ratio was maintained for $1.5 \mathrm{~min}$. The column was re-equilibrated with $10 \% \mathrm{~B}$ for $1 \mathrm{~min}$. The APCI ionization operated at a discharge current of $5 \mu \mathrm{A}$, a vaporizer temperature of $350{ }^{\circ} \mathrm{C}$ and a capillary temperature of $200{ }^{\circ} \mathrm{C}$. Compound purity was determined using the ratio of the appropriate peak area to sum of areas of all peaks of the mixture. Areas were determined by integration of the peaks from the PDA detector response. Purity of final compounds was determined by this method and was $>95 \%$.

Purification was performed using semi-preparative HPLC with a Waters 1500 series HPLC equipped with a 2707 Autosampler, a 1525 binary HPLC pump, a 2998 Waters Photodiode Array Detector and a Waters Fraction Collector III with a YMC C18 reverse phase column $(20 \times 100 \mathrm{~mm}, 5 \mu \mathrm{m}$ particle size $)$. The mobile phase consisted of acetonitrile and a $10 \mathrm{mM}$ aqueous ammonium acetate gradient over $6 \mathrm{~min}$.

NMR spectra were measured in $\mathrm{CDCl}_{3}$ or DMSO- $\mathrm{d}_{6}$ using a Jeol ECX-500 (500 MHz) spectrometer. Chemical shifts $(\delta)$ are reported in parts per million (ppm), and coupling constants $(J)$ are reported in Hertz $(\mathrm{Hz})$.

HR-MS analysis was performed using an Orbitrap Elite highresolution mass spectrometer (Thermo Fischer Scientific, MA, USA) operating at positive full scan mode (120 $000 \mathrm{FWMH})$ in the range of 2000-3000 $\mathrm{m} / \mathrm{z}$. The settings for electrospray ionization were as follows: oven temperature of $300{ }^{\circ} \mathrm{C}$, sheath gas of 8 arb. units and source voltage of $1.5 \mathrm{kV}$. Samples were diluted to a final concentration of $20 \mu \mathrm{mol} \mathrm{l}^{-1}$ with $0.1 \%$ formic acid in water and methanol (50:50, v/v).

Rink amide resin and Fmoc-amino acids were purchased from AAPPTec (Louisville, KY). Solvents and other chemicals were purchased from Sigma-Aldrich (Milwaukee, IL, http:// www.sigmaaldrich.com).

Fluorescence spectra were recorded on a Varian Cary Eclipse fluorescence spectrophotometer equipped with a thermostat (FL1009M015). Excitation and emission slits were $5 \mathrm{~nm}$. Absorption spectra were recorded on a Cary $300 \mathrm{UV} / \mathrm{Vis}$ spectrophotometer (UV111M031, Agilent). Excitation spectra of the model drugs 1-3 correspond to general observations published previously $^{27,28}$ and don't interfere with the spectra of the conjugates 4-6 or released dye 7 .

\section{Quantum yield determination}

Quantum yields $(\Phi)$ were calculated by standard procedure using Fluorescein as a reference $(\Phi=0.91)$ according to eqn $(1)$.

$$
\Phi=\Phi_{\mathrm{R}} \times I / I_{\mathrm{R}} \times A_{\mathrm{R}} / A \times \eta^{2} /\left(\eta_{\mathrm{R}}{ }^{2}\right)
$$

where $\Phi_{\mathrm{R}}$ is the quantum yield of reference fluorophore, $I$ is area under emission peak, $A$ is absorbance at the excitation wavelength $\eta$ is refractive index of the solvent.

\section{Cleavage of the conjugates 4-6 by glutathione and its LC/MS monitoring}

$0.25 \mathrm{ml}$ of the conjugate 4,5 and 6 solution $(2 \mathrm{mM})$ in DMSO was mixed with $0.1 \mathrm{ml}$ of GSH solution $(50 \mathrm{mM})$ in HEPES (0.1 M, pH 7.4) and diluted with $0.65 \mathrm{ml}$ DMSO/HEPES (2: $1 \mathrm{v} /$ v). The mixture was heated to $37{ }^{\circ} \mathrm{C}$ and analyzed by LC/MS within the time. Intracellular cleavage of conjugates 4-6 by glutathione and its fluorescence monitoring.

\section{Cleavage of the conjugates 4-6 by glutathione and its fluorescence monitoring}

$5 \mu$ of the probe 4,5 or 6 solution $(1 \mathrm{mM})$ in DMSO was mixed with $20 \mu \mathrm{l}, 60 \mu \mathrm{l}$ or $100 \mu \mathrm{l}$ of the GSH solution (50 mM) in HEPES buffer (0.1 M; pH 7.4) and diluted by HEPES buffer $(0.1 \mathrm{M} ; \mathrm{pH}$ 7.4) or DMSO/HEPES buffer $(2: 1)$ to $1 \mathrm{ml}$. The mixture was heated to $37^{\circ} \mathrm{C}$ and the fluorescence was measured within the time.

\section{Intracellular cleavage of conjugates $4-6$ by glutathione and its fluorescence monitoring}

HeLa cells were added to black 96-well plates by MultiDrop Combi (Thermo Fisher Scientific, USA) at a cell density of $1.25 \times$ $10^{4}$ per well and incubated overnight. Pre-treatment with GSH was performed by incubation of cells with GSH $(20 \mathrm{mM}$ in medium) for $2 \mathrm{~h}$. The cells were washed with PBS, immediately treated with tested compounds for 2 min and washed with PBS again. Finally, $50 \mu \mathrm{l}$ of PBS was added to each well. Fluorescence intensity was measured by EnVision plate reader (PerkinElmer, USA), two reads for each time point (first with ex $510 \mathrm{~nm} / \mathrm{em}$ $535 \mathrm{~nm}$ and second with ex $485 \mathrm{~nm} / \mathrm{em} 535 \mathrm{~nm}$ ). 


\section{Fluorescent microscopy imaging}

HeLa cells (3000 per well, $30 \mu \mathrm{l}$ per well) were seeded into 384 CellCarrier plates (PerkinElmer, USA) for live cell fluorescence imaging and preincubated for $24 \mathrm{~h}$ at $37{ }^{\circ} \mathrm{C}$ and $5 \% \mathrm{CO}_{2}$ to adhere. The cells were pretreated with $20 \mathrm{mM} \mathrm{GSH}$ for $2 \mathrm{~h}$ and stained by Hoechst dye in concentration $1.62 \mu \mathrm{M}$ within last $20 \mathrm{~min}$. Further, the stained cells were rinsed with fresh media, treated with tested compounds $(10 \mu \mathrm{M})$ for $2 \mathrm{~min}$ and rinsed with fresh media again. The live-cell imaging was performed by Cell Voyager CV7000 (Yokogawa, Japan) spinning disc confocal microscopy system at $37{ }^{\circ} \mathrm{C}$ in $5 \% \mathrm{CO}_{2}$ atmosphere. Living cells were monitored by a $40 \times$ water immersion objective. All images were post-processed, background subtracted and deconvolved using Image J software.

\section{Results and discussion}

This novel system is suggested as the conjugate of aminoBODIPY dye acylated by (a) symmetrical self-immolative disulfide linker, connected to a drug predestinated to release. This system was designed based on the previous study by Jain et al. ${ }^{29}$ The symmetrical linker can be used for binding of a drug with an amino or hydroxy group, whereas the asymmetrical linker can be used for binding of a drug via its thiol group (Scheme 1). The disulfide bridge provides a switch that triggers the release of the drug and free amino-BODIPY in the presence of thiols.

To study the possibility of monitoring a drug release, we used the compounds 1-3 from a group of 2-phenyl-3-hydroxy$4(1 H)$-quinolinone derivatives, known for their anticancer activity $^{\mathbf{3 0}}$ as the model drugs. These drugs are substituted by the thiol, hydroxy, or amino group, suitable for conjugation with amino-BODIPY 7. The structure of these model drugs and their conjugates 4-6 are presented in Fig. 1.

The model drug 1 was synthesized from quinolinone 8 (ref. 30) by standard peptide synthesis with immobilized Fmoccysteine on Rink resin. Compound 2 was prepared by esterification of the starting derivative $\mathbf{9}$ with triethyleneglycol (Scheme 2). Compound 3 was synthesized according to the previously published procedure. ${ }^{30}$

Amino-BODIPY 7 was prepared by reaction of previously published chloroderivative $\mathbf{1 0}$ (ref. 31) with ammonia in

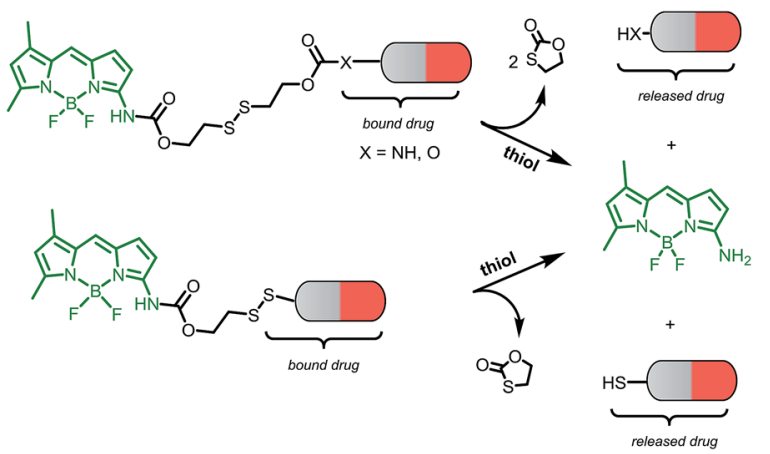

Scheme 1 Schematic illustration of the drug and amino-BODIPY release from their conjugates.

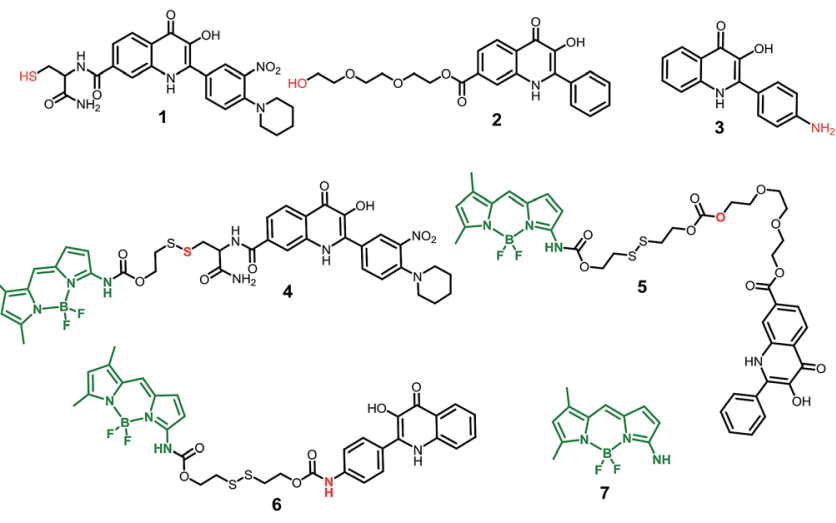

Fig. 1 Structure of model drugs 1, 2, and 3 (ref. 18) and their conjugates 4,5 , and 6 used for thiol-mediated cleavage studies.

methanol/DCM as described previously. The reaction with disulfide precursor $\mathbf{1 1}$ (ref. 32) effectuated the intermediate $\mathbf{1 2}$. The disulfide exchange of this intermediate with mercapto derivative 1 afforded the final disulfide conjugate 4 (Scheme 3).

The coupling of amino-BODIPY 7 with linker precursor 13 and subsequent reaction with HOBt gave rise to compound $\mathbf{1 4}$ with activated carbonate group. Then, final conjugates 5 and 6 were prepared by coupling the derivative $\mathbf{1 4}$ with the corresponding quinolinones 2 or 3 respectively via carbamate or carbonate bond (Scheme 3).

The fluorescence spectra of amino-BODIPY 7 and its conjugates 4-6 were first measured in HEPES buffer $(0.1 \mathrm{M}, \mathrm{pH} 7.4)$ to meet the requirements for the planned biological experiments.

Amino-BODIPY 7 has one broad excitation maximum at $480 \mathrm{~nm}$ and emission at $523 \mathrm{~nm}$ (Table 1; Fig. 2). AcylaminoBODIPY in conjugates 4-6 possesses excitation maxima 515$517 \mathrm{~nm}$, while emission of $525-527 \mathrm{~nm}$ is very close to the emission of amino-BODIPY 7 (Fig. 2; Table 1).

Besides the shift of excitation maxima, a significant difference was observed between amino-BODIPY 7 and its conjugates 4-6 with respect to the quantum yields and fluorescence intensity. When excitation wavelength at $480 \mathrm{~nm}$, which is the characteristic maximum for amino-BODIPY 7, was used and emission at $525 \mathrm{~nm}$ was collected, a significant difference was observed between the fluorescence intensity of amino-BODIPY 7 and its conjugates 4-6 (Fig. 3), and thus, the system can work as OFF-ON during the cleavage. The intensity ratio of aminoBODIPY 7 to appropriate conjugate 4-6 differs probably due to different quenching effect of the bound derivatives 1-3.

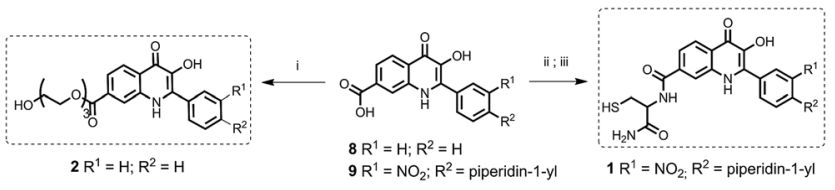

Scheme 2 Synthesis of model drugs 1 and 2. (i) 8, triethyleneglycol, $\mathrm{H}_{2} \mathrm{SO}_{4}$ (cat.), THF, reflux, on; (ii) Rink amide resin preloaded with cysteine, 9, HOBt, DIC, DMF/Pyr, 3 h, rt. (iii) TFA/DCM/TES (2 : $1: 0.05$ ), $1 \mathrm{~h}, \mathrm{rt}$. 


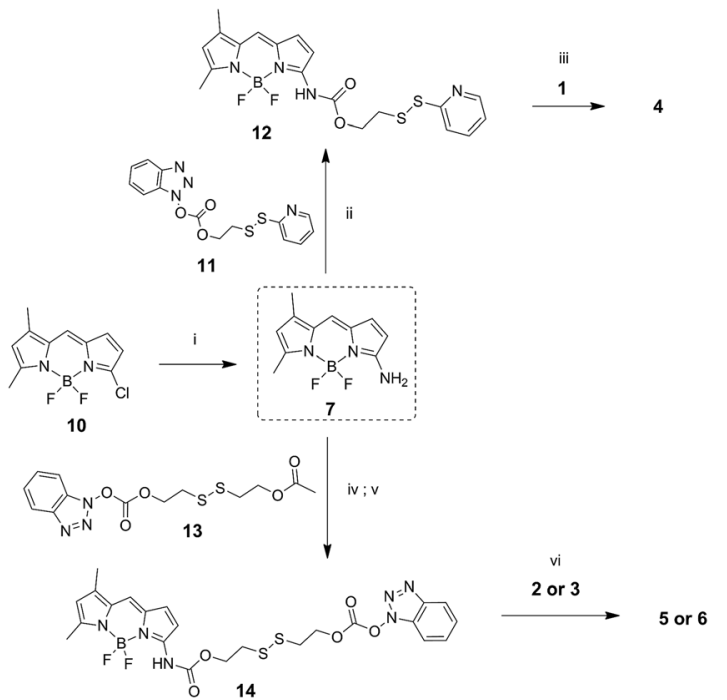

Scheme 3 Synthesis of amino-BODIPY 7 and its conjugates 4-6. (i) 7.0 $\mathrm{M} \mathrm{NH}_{3} / \mathrm{MeOH}, \mathrm{DCM} 70{ }^{\circ} \mathrm{C}$, on; (ii) DMAP, TEA, DCM, rt, $3 \mathrm{~h}$; (iii) DMF, $60^{\circ} \mathrm{C}$, on; (iv) TEA, DMAP, $13, \mathrm{DCM}, \mathrm{rt}, 3 \mathrm{~h}$. (v) $\mathrm{K}_{2} \mathrm{CO}_{3}, \mathrm{THF} / \mathrm{MeOH}$ $3: 1$, rt, on; (vi) DMAP, TEA, DCM, rt, on.

Therefore a significant difference was observed between aminoBODIPY 7 and conjugate 4 , in which the fluorescence is expectedly quenched by the nitro group.

When the excitation wavelength was selected at $510 \mathrm{~nm}$, what is close to the excitation maxima of conjugates 4-6 and sufficiently distal from the emission maximum of BODIPY 7 , the emission intensity was high for amino-BODIPY 7 due to its broad excitation spectrum and high quantum yield (Fig. 3; Table 1). The system could work as OFF-ON during the cleavage as well, but less efficiently.

To assess the effect of solvent polarity to OFF-ON effect, we mixed the HEPES buffer with DMSO $(1: 2 \mathrm{v} / \mathrm{v})$. Based on the results from Table 1 and Fig. 4, the excitation as well as emission spectra of conjugates 4-6 exhibit bathochromic shift to approximately $520 \mathrm{~nm}$ and $530 \mathrm{~nm}$, respectively and enhanced quantum yield.

Table 1 Fluorescence profile of conjugates 4-6, 18, 19 and BODIPY dyes 7,20

\begin{tabular}{llllll}
\hline Compound & Solvent & $\lambda_{\text {exc }}(\mathrm{nm})$ & $\lambda_{\text {em }}(\mathrm{nm})$ & $\Delta \lambda(\mathrm{nm})$ & QY $(\%)$ \\
\hline $\mathbf{4}$ & HEPES & 515 & 527 & 12 & 1.4 \\
& DMSO/HEPES & 517 & 531 & 14 & 7.7 \\
$\mathbf{5}$ & HEPES & 516 & 525 & 9 & 14 \\
& DMSO/HEPES & 520 & 530 & 10 & 58 \\
$\mathbf{6}$ & HEPES & 517 & 525 & 8 & 32 \\
7 & DMSO/HEPES & 521 & 532 & 11 & 53 \\
& HEPES & 480 & 523 & 43 & 77 \\
$\mathbf{1 9}$ & DMSO/HEPES & 496 & 523 & 40 & 95 \\
$\mathbf{2 0}$ & DMSO/HEPES & 521 & 549 & 28 & 23 \\
& DMSO/HEPES & 520 & 531 & 11 & 95 \\
& DMSO/HEPES & 521 & 550 & 29 & 48
\end{tabular}

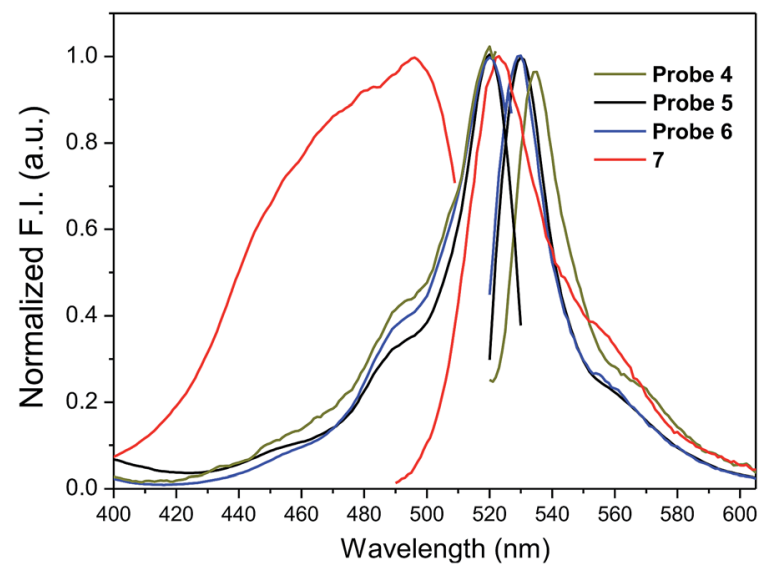

Fig. 2 Normalized fluorescence excitation and emission spectra of amino-BODIPY 7 and its conjugates 4-6 (HEPES buffer, $0.1 \mathrm{M}, \mathrm{pH}$ 7.4).

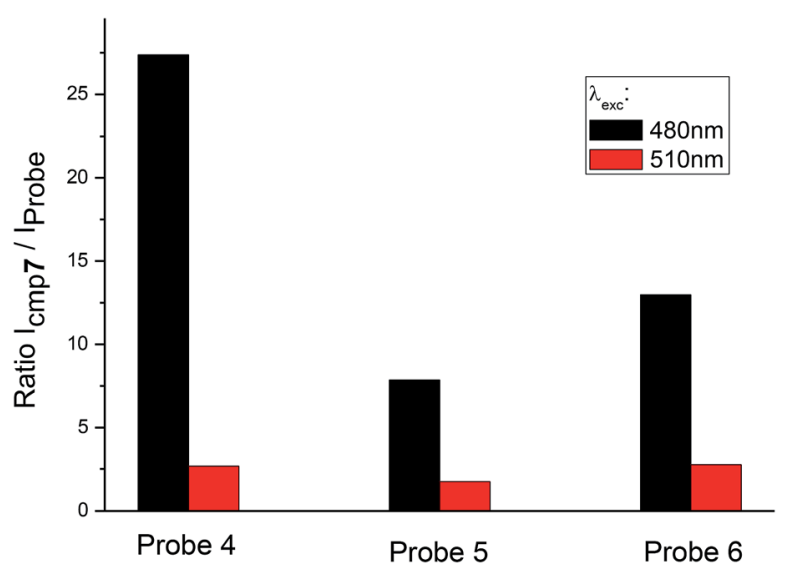

Fig. 3 Ratio of fluorescence intensity of amino-BODIPY 7 to conjugates $4-6$ at $525 \mathrm{~nm}$ after excitation at $480 \mathrm{~nm}$ or $510 \mathrm{~nm}$ (HEPES buffer, $0.1 \mathrm{M}, \mathrm{pH} 7.4$ ).

When the excitation wavelength $\lambda_{\text {exc }}=480 \mathrm{~nm}$ is applied, the emission intensity ratio for $\lambda_{\mathrm{em}}=530 \mathrm{~nm}$ between aminoBODIPY 7 and its conjugates 4-6 is approximately $2-3$, and

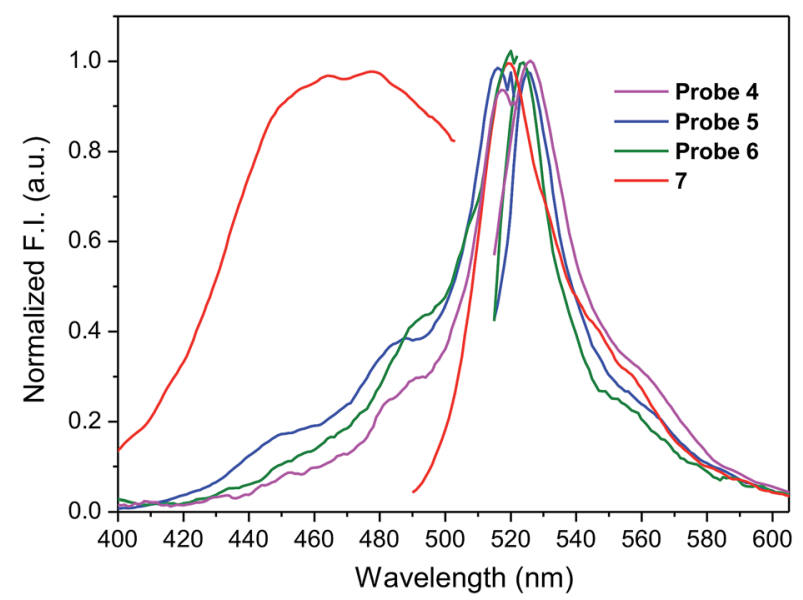

Fig. 4 Fluorescence excitation and emission spectra of aminoBODIPY 7 and conjugates 4-6 (DMSO/HEPES buffer 2 : 1, 0.1 M, 7.4 $\mathrm{pH})$. 


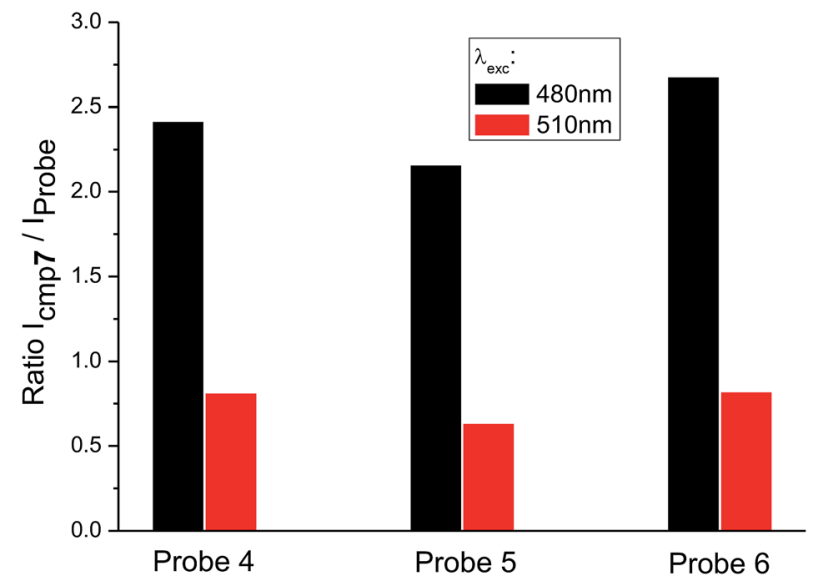

Fig. 5 Ratio of fluorescence intensity of amino-BODIPY 7 to conjugates 4-6 at $530 \mathrm{~nm}$ after excitation of the appropriate couple at $480 \mathrm{~nm}$ or $510 \mathrm{~nm}$ (DMSO/HEPES buffer $2: 1,0.1 \mathrm{M}, \mathrm{pH} 7.4$ ).

hence, the OFF-ON effect is not as effective as in the HEPES buffer. When $\lambda_{\text {exc }}=510 \mathrm{~nm}$ is used, the fluorescence intensity is lower for amino-BODIPY 7 as compared to conjugates 4-6 (Fig. 5).

To confirm the ability of disulfide linker to release the amino-BODIPY 7 and the model drugs 1-3 by thiols according to Scheme 1, conjugates 4-6 were treated with $0.5 \mathrm{mM}$ glutathione at physiological conditions $\left(37{ }^{\circ} \mathrm{C}, \mathrm{pH} 7.4\right)$ and monitored by LC/MS. Because of the necessity to use a high concentration of the probes for such a detection, the present study was performed only in DMSO/HEPES buffer $(2: 1)$. As shown in Fig. 6, the treatment of prodrug 4 with GSH resulted in four products detected by HPLC. According to mass spectrometry, we detected the presence of the expected released drug 1 and amino-BODIPY 7 indicating complete cleavage of the conjugate, but also the formation of adducts $\mathbf{1 5}$ and 16 derived from the 3-HQ or BODIPY dye. Interestingly, the concentration of the adduct $\mathbf{1 5}$ increases in a time-dependent manner, which might be attributed to the equilibrium between 15 and 1 . Conversely, the concentration of adduct $\mathbf{1 6}$ decreased with time because of the presence of self-immolative disulfide linker that prevents similar equilibrium.

The cleavage of the conjugates 5 and $\mathbf{6}$, wherein $3 \mathrm{HQ}$ is bound via carbonate and carbamate bonds, afforded the corresponding 3HQs 2 and 3, respectively. Although the GSH adducts 16 and 17 were also observed, these were subsequently converted to the final free $3 \mathrm{HQ}$ derivative 2 or $\mathbf{3}$ and aminoBODIPY 7 (Fig. 6B and C). The treatment using all prodrugs 4-6 was performed with $20 \mu \mathrm{M}$ in the extracellular matrix. ${ }^{33}$ No cleavage was observed for any derivative in this case.

The difference in fluorescence profile of amino-BODIPY 7 and conjugates 4-6 should enable efficient monitoring of the cleavage of conjugates via OFF-ON mode in HEPES buffer (Fig. 3), which is optimal for potential biological applications. Next we performed the GSH-mediated cleavage of probes 4-6 monitored by fluorescence with excitation at $480 \mathrm{~nm}$ as well as $510 \mathrm{~nm}$ and emission at $525 \mathrm{~nm}$. Within $180 \mathrm{~min}$ we observed the expected enhancement of the fluorescence intensity for excitation at $480 \mathrm{~nm}$ and $510 \mathrm{~nm}$ (Fig. 7; Fig. S2 and S4 $\dagger$ ).

Furthermore, plotting the ratio of emission intensities at $525 \mathrm{~nm}$ after excitation at $480 \mathrm{~nm}$ and $510 \mathrm{~nm}\left(I_{480} / I_{510}\right) v s$. time allows ratiometric fluorescence monitoring the cleavage in a concentration independent manner (Fig. 8).

The rate of the GSH-mediated cleavage of disulfide bridge depends on the concentration of GSH. While $5 \mathrm{mM}$ GSH normally present in some cancer cells, ${ }^{\mathbf{1 4}}$ was sufficient for full cleavage of conjugate 4 within $50 \mathrm{~min}$ and $120 \mathrm{~min}$ for conjugates 5 and $\mathbf{6}$, respectively; the low concentrations of GSH caused only partial cleavage during the same period (Fig. 8). The limit of detection (LOD) for GSH was determined for compounds 5 and 6 (HEPES buffer $7.4 \mathrm{pH}, 37{ }^{\circ} \mathrm{C}, 2 \mathrm{~h}$ incubation). Obtained LOD values were $305 \mathrm{nM}$ and $752 \mathrm{nM}$, respectively (ESI, $\uparrow$ Fig. 12). In addition, the cleavage is $\mathrm{pH}$ dependent and proceeds rapidly in basic conditions. In acidic medium, none or only little conjugate is cleaved (Fig. 9).

As exemplified on probe 4 not only GSH but also cysteine, commonly present in a biological medium, can cause the amino-BODIPY 7 releasing. On the other hand, the system is resistant to all other amino acids (Fig. 10).

The release of amino-BODIPY 7 from conjugates 4-6 is accompanied by the release of drugs 1-3 (Fig. 6) and therefore enhancing the fluorescence of amino-BODIPY 7 reflects release of the drugs. Thus, the monitoring of the drug release is dependent only on the change of acylamino-BODIPY to aminoBODIPY derivative and is independent of the type of the drug. To prove this hypothesis, compounds 18, 19, and 20 with different substitutions on amino-BODIPY (Fig. 11) were synthesized as described in Scheme 3 (see ESI $\dagger$ ). Conjugate 18 and released BODIPY dye 20 showed the same excitation/ emission profile due to the same piperidyl substitution directly bound to the BODIPY scaffold. On the other hand, conjugate 19 effectuating amino-BODIPY 7 after cleavage follows the same excitation/emission profile changes as conjugates 4-6 (Fig. 11 and 4). Thus, we can conclude that the principle of OFF-ON effect is not influenced by the nature of a compound conjugated to BODIPY dye, but rather depends only on the transformation of acylamino-BODIPY to aminoBODIPY derivative.

Probe 4-based monitoring of the cleavage by UV/Vis spectroscopy resulted in the hyperchromic as well as hypochromic shift with an isosbestic point at approximately $490 \mathrm{~nm}$ (Fig. 12). As the absorption profile of quinolinones 1-3 bound in conjugates 4-6 did not interfere with this wavelength (see ESI $\dagger$ ), the isosbestic point was connected with the change of acylaminoBODIPY to amino-BODIPY regardless of the bound drug. This phenomenon could be supported by the same results from UV/ Vis-based monitoring of cleavage of conjugate 19 bearing cysteine having no absorption in the UV/Vis region (Fig. 12).

The developed system might be used for monitoring the release of a drug inside the cancer cells, well known for the increased level of redox potential due to high concentration of thiols, mainly GSH. ${ }^{\mathbf{1 4 , 1 5}}$ A maximal release of a drug within a time period corresponded to the maximal release of aminoBODIPY 7 from appropriate conjugate (Scheme 1; Fig. 6). To 

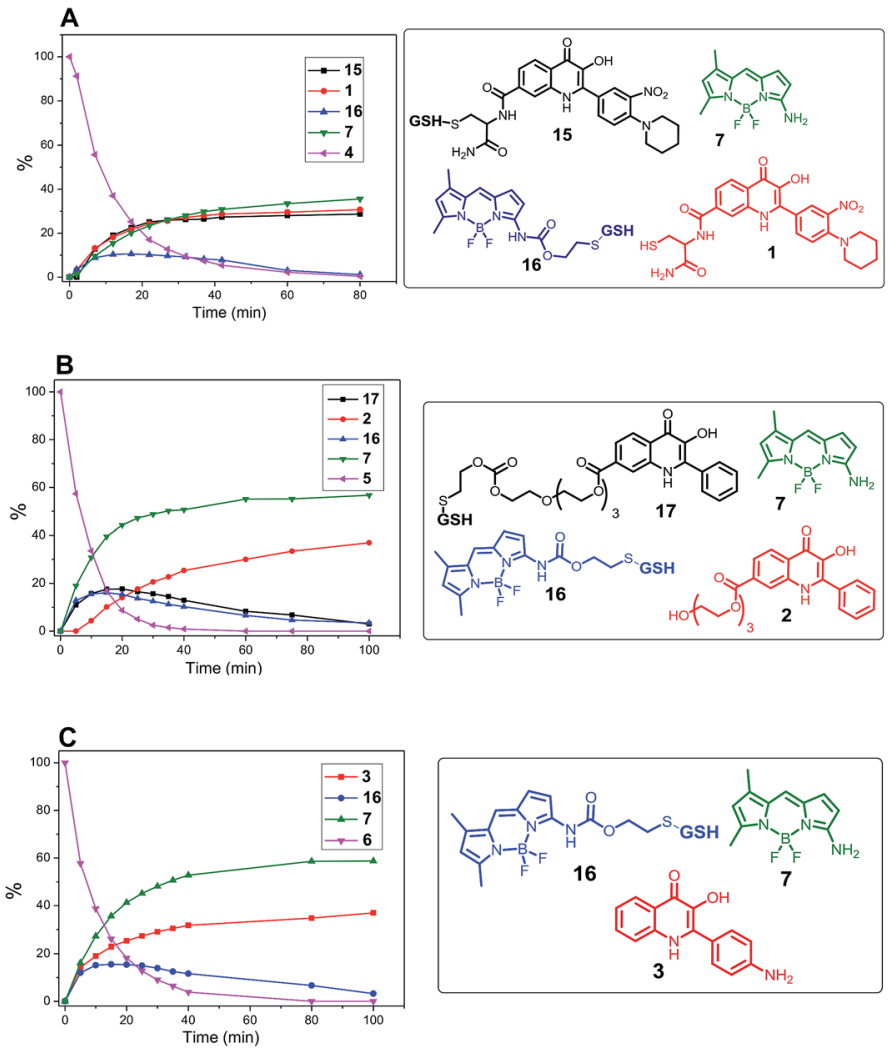

Fig. 6 Monitoring the drug release from conjugates 4,5 , and $6\left(0.5 \mathrm{mM}\right.$; DMSO/HEPES buffer $\left.2: 1,0.1 \mathrm{M} ; \mathrm{pH} 7.4 ; 37^{\circ} \mathrm{C}\right)$ by GSH $(5 \mathrm{mM})$ by HPLC/ MS.

prove the real functioning of the system, we performed the cleavage experiment of conjugates 4-6 in HeLa cells that were pretreated with glutathione $(20 \mathrm{mM})$. Due to the instrumental
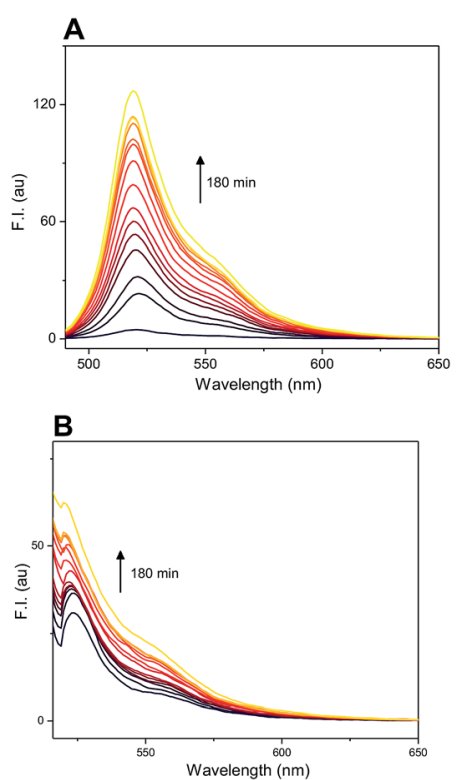

Fig. 7 GSH-mediated cleavage of probe 4 monitored within $180 \mathrm{~min}$ by fluorescence emission at $525 \mathrm{~nm}$ after excitation at $480 \mathrm{~nm}(\mathrm{~A})$ and $510 \mathrm{~nm}(\mathrm{~B})(5 \mu \mathrm{M}$ probe 4, $5 \mathrm{mM}$ GSH; HEPES buffer, $0.1 \mathrm{M}, \mathrm{pH} 7.4,37$ $\left.{ }^{\circ} \mathrm{C}\right)$. limitation, excitation at $485 \mathrm{~nm}$ was used instead of $\lambda_{\text {exc }}=$ $480 \mathrm{~nm}$. Although the inherent concentration of GSH in the cells was sufficient to cleave the disulfide conjugates $4-\mathbf{6}$, the
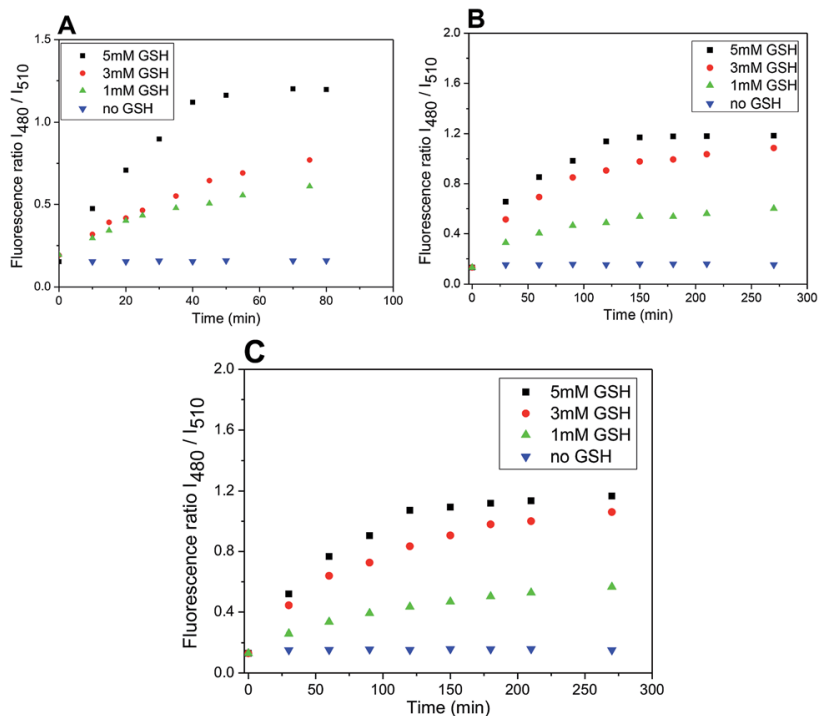

Fig. 8 Ratio of emission intensities at $525 \mathrm{~nm}$ upon excitation at 480 and $510 \mathrm{~nm}$ in time after treatment with different concentrations of GSH $(5,3,1 \mathrm{mM})$. Probe 4 (A); probe 5 (B); probe 6 (C) $(5 \mu \mathrm{M}$ probes $4-$ 6; DMSO/HEPES buffer $2: 1,0.1 \mathrm{M}, \mathrm{pH} 7.4,37^{\circ} \mathrm{C}$ ). 

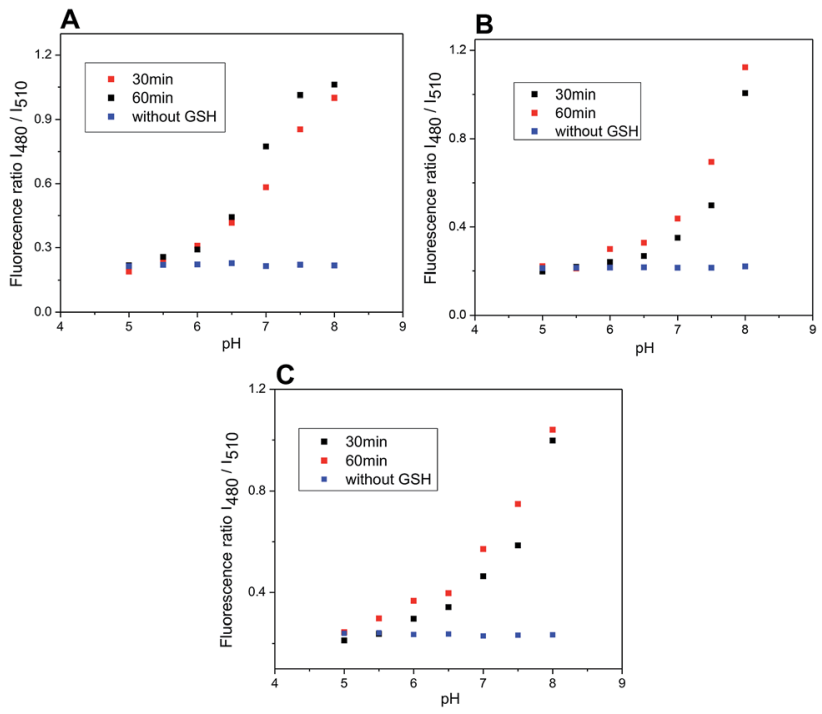

Fig. 9 Ratio of emission intensities at $525 \mathrm{~nm}$ upon excitation at 480 and $510 \mathrm{~nm}$ after 30 and 60 minutes of incubation of probes 4-6 with GSH at various $\mathrm{pH}(5.0-8.0)$ and without GSH after 60 minutes. Probe $4(\mathrm{~A})$; probe $5(\mathrm{~B})$; probe 6 (C) $(5 \mu \mathrm{M}$ probe $4-6,5 \mathrm{mM}$ glutathione; DMSO/HEPES buffer $2: 1,0.1 \mathrm{M}, 37^{\circ} \mathrm{C}$ ).

cleavage was efficient when conjugates were added to the cells preincubated with additional GSH (Fig. 13), which is similar to the previous results (Fig. 8). The exception is conjugate 5 with the most susceptible carbonate linker between dye and model drug. Supposedly, the GSH concentration in HeLa cells is sufficiently high for maximal cleavage of the linker, and the preincubation with GSH does not accelerate the disruption of the disulfide bond.

The release of a drug inside the cells can be monitored via the OFF-ON effect by fluorescence microscopy. For this purpose, the HeLa cells were localized by staining the nuclei by Hoechst dye (blue color) and then treated with the conjugate 4, generating green emission due to the release of amino-BODIPY

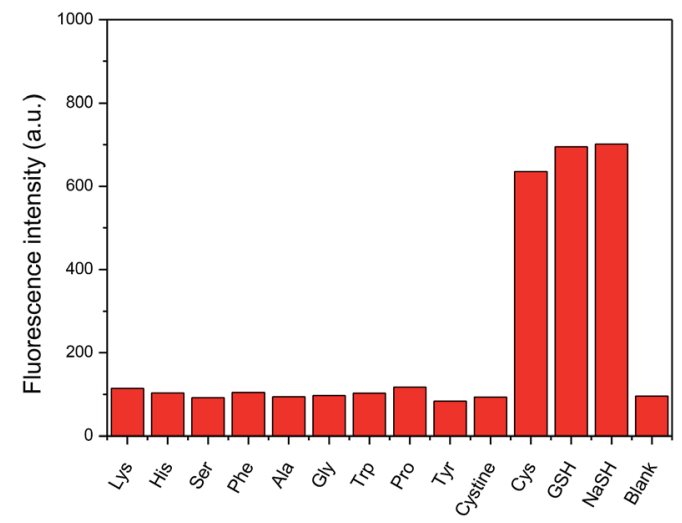

Fig. 10 Fluorescence intensity enhancement upon incubation of probe $4(5 \mu \mathrm{M})$ with various amino acids and NaSH $(100 \mathrm{mM}$, DMSO/ HEPES buffer $2: 1,0.1 \mathrm{M}, \mathrm{pH} 7.4)$ after $60 \mathrm{~min}$. $\left(\lambda_{\mathrm{exc}}=480 \mathrm{~nm}, \lambda_{\mathrm{em}}=\right.$ $525 \mathrm{~nm})$
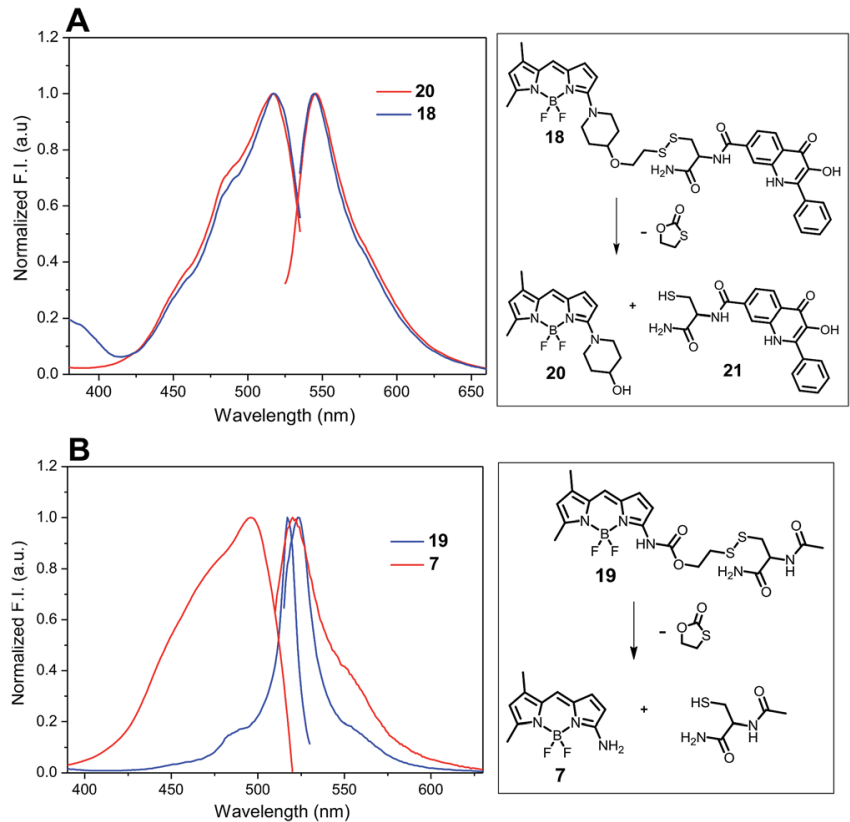

Fig. 11 Normalized excitation and emission profile of compounds 18 , 19 , and $20(5 \mu \mathrm{M}$ in DMSO/HEPES buffer $2: 1,0.1 \mathrm{M}, \mathrm{pH} 7.4)$.

7 and the corresponding drug in a time-dependent manner (Fig. 14).

The other possible applicability of the introduced system can be demonstrated by the construction of a molecular electronic "selector" of two sources for one appliance. Therefore, the derivative 4 was studied in DMSO/HEPES $(2: 1 \mathrm{v} / \mathrm{v})$ at a concentration of $5 \mu \mathrm{M}$. If none or only one of the abovementioned conditions (lack of thiols and $\mathrm{pH}<6$ ) were fulfilled, the conjugate was stable (Fig. 9). However, when both conditions were fulfilled, the conjugate was cleaved. In the case of intensity of emission at $527 \mathrm{~nm}$ or $543 \mathrm{~nm}$, the excitation at $\lambda_{\text {exc }}=510 \mathrm{~nm}$ before cleavage and at $\lambda_{\text {exc }}=480 \mathrm{~nm}$ after cleavage was similar (Fig. 15).

The molecular selector represented schematically in Fig. 16, wherein two circuits with different power supplies are connected to one light. The selector able to change the source is operated by the tandem of molecular gates AND and NAND operated by thiol and $\mathrm{pH}$ inputs. Before the conjugate cleavage
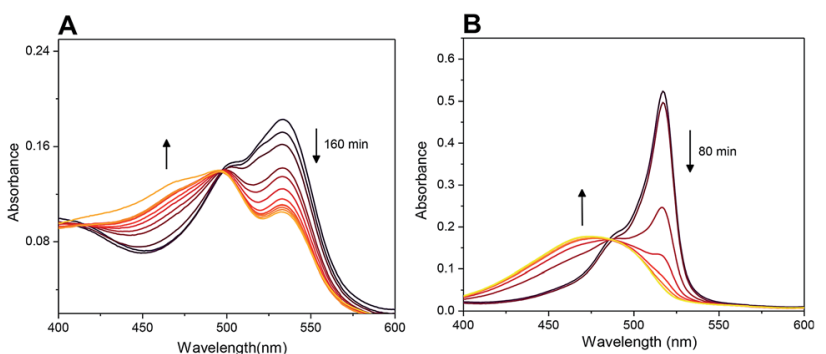

Fig. 12 Monitoring of cleavage of probes 4 (A) and 19 (B) by UV/Vis spectroscopy ( $5 \mu \mathrm{M}$ probes; $5 \mathrm{mM}$ GSH, 0.1 M HEPES buffer, $\mathrm{pH} 7.4,37$ ${ }^{\circ} \mathrm{C}$ ). For spectra highlighting change of fluorescence in time see Fig. 7 and S7.† 

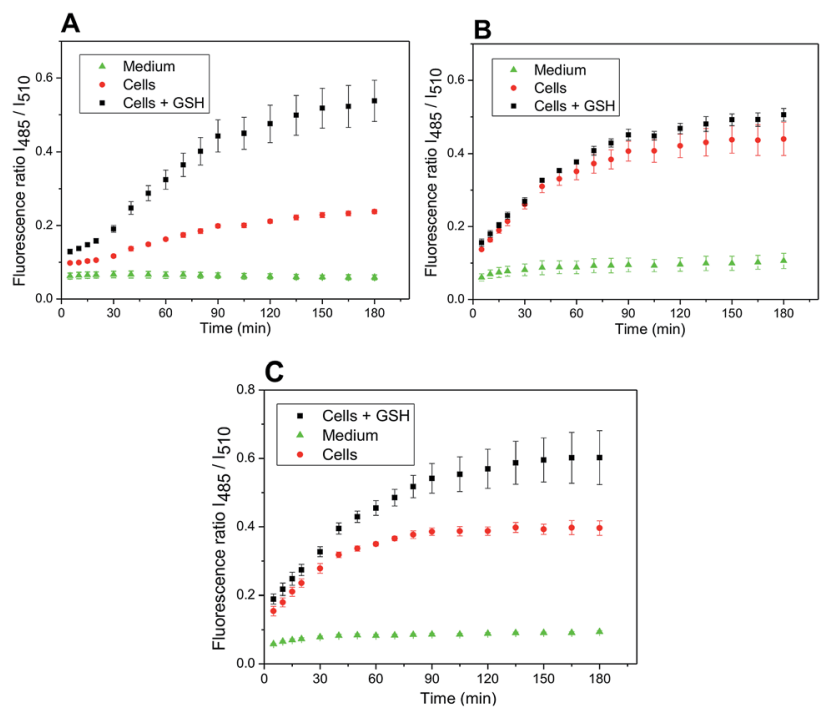

Fig. 13 Ratio of emission intensities at $525 \mathrm{~nm}$ upon excitation at $485 \mathrm{~nm}$ and $510 \mathrm{~nm}$ in HeLa cells corresponding to the cleavage of conjugate $4(\mathrm{~A}), 5(\mathrm{~B})$, and $6(\mathrm{C})$ within the prescribed period.
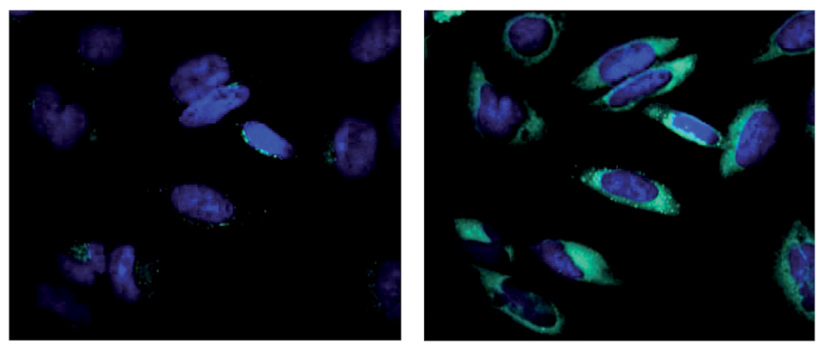

Fig. 14 Fluorescence intensity of released amino-BODIPY 7 (green color) after cleavage of conjugate 4 inside the HeLa cells pretreated with glutathione at time $0 \mathrm{~min}$ (left) and $120 \mathrm{~min}$ (right). $\lambda_{\text {exc }}=485 \mathrm{~nm}$.

the output of operator NAND is "one" and source $520 \mathrm{~nm}$ is active. When the conjugate is cleaved NAND operator has the output "zero" and immediately operator AND produces output

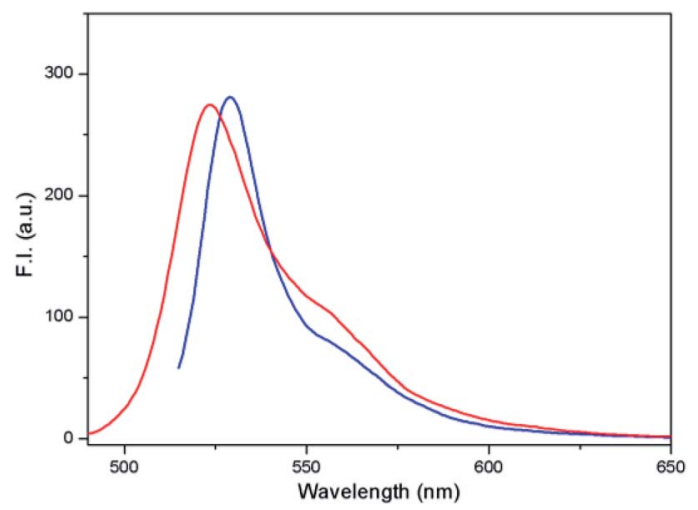

Fig. 15 Fluorescence emission spectra of compound 4 ( $5 \mu \mathrm{M}$; DMSO/ HEPES buffer $2: 1,0.1 \mathrm{M}, \mathrm{pH} 7.4$ ) after excitation at $510 \mathrm{~nm}$ (blue) before cleavage and at $480 \mathrm{~nm}$ (red) after cleavage.

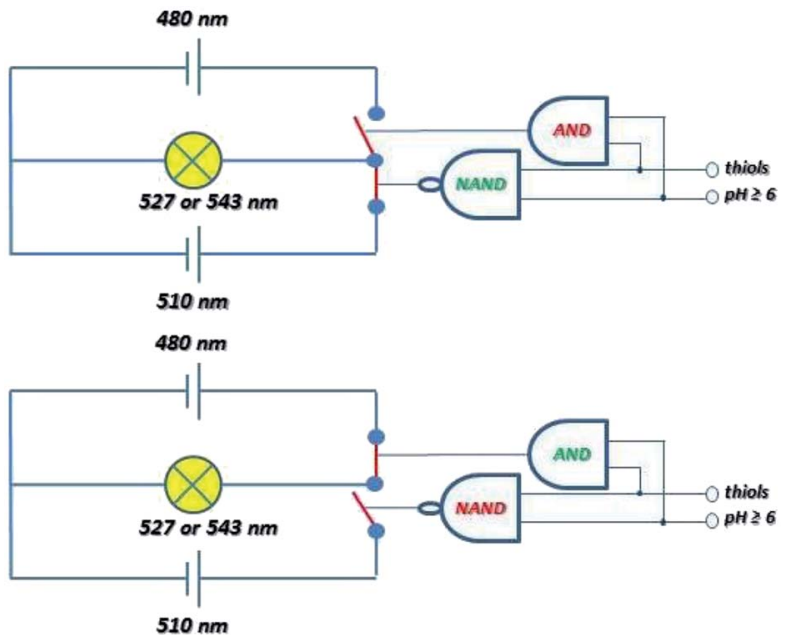

\begin{tabular}{|cccc|}
\hline Thiols & $\mathrm{pH} \geq 6.5$ & NAND & AND \\
\hline 0 & 0 & 1 & 0 \\
\hline 0 & 1 & 1 & 0 \\
\hline 1 & 0 & 1 & 0 \\
\hline 1 & 1 & 0 & 1 \\
\hline
\end{tabular}

Fig. 16 Schematic description of the selector based on cleavage of probe 4 able to switch the source of the light to maintain the same intensity and accuracy based on the truth table.

"one. The source $480 \mathrm{~nm}$ is than active. This circuitry imitates "emergency power unit" for the generation of energy for the "bulb" after the failure of the main source.

\section{Conclusion}

In conclusion, the conjugates of amino-BODIPY and model drugs bound by self-immolative linker were synthesized and analyzed using glutathione for their cleavage with respect to the change in fluorescent properties. The spectral differences between acylamino-BODIPY in the conjugates and aminoBODIPY released after the glutathione attack enabled the monitoring of the release based on two excitations/one emission ratiometric fluorescence or via the OFF-ON effect. Thus, the drug release corresponded to the release of the aminoBODIPY used for monitoring, rendering the system valuable for monitoring the release of non-fluorescent drugs. Moreover, the drug interactions are effectuated via their amino, hydroxy, or thiol group that renders versatility to the compound structure. The rate of drug release is dependent on the concentration of glutathione as well as on the $\mathrm{pH}$ of the solution. The monitoring of the model drug release via ratiometric fluorescence as well as the OFF-ON effect was also verified in cancer cells with native and artificially increased concentration of glutathione. Furthermore, the UV/Vis spectroscopy allows the estimation of the concentration of conjugates independently on the extent of cleavage. 
The altered excitation wavelengths post-cleavage also showed similar emission, and hence, was used for the construction of molecular electronic selector. This selector was operated by two logic gates for irreversible switching of the two sources to maintain the intensity of one light.

The concept of acylamino-BODIPY conjugates affording the amino-BODIPY dye after thiol-mediated cleavage based on the altered fluorescence offers development of various applications in chemical biology and molecular electronics in the future.

\section{Conflicts of interest}

There are no conflicts to declare.

\section{Acknowledgements}

This work was supported by the National Program of Sustainability (project LO1304) and by Technology Agency of the Czech Republic (project TE02000058).

\section{References}

1 Z.-R. Lu and P. Qiao, Mol. Pharm., 2018, 15, 3603-3616.

2 S. S. Kelkar and T. M. Reineke, Bioconjugate Chem., 2011, 22, 1879-1903.

3 T. Kowada, H. Maeda and K. Kikuchi, Chem. Soc. Rev., 2015, 44, 4953-4972.

4 D. Zhang, J. R. Cochrane, A. Martinez and G. Gao, RSC Adv., 2014, 4, 29735-29749.

5 S. Shanmugaraju and P. S. Mukherjee, Chem. Commun., 2015, 51, 16014-16032.

6 T. Rasheed, C. Li, M. Bilal, C. Yu and H. M. N. Iqbal, Sci. Total Environ., 2018, 640-641, 174-193.

7 W. Meng, Y. Chen, Y. Feng, H. Zhang, Q. Xu, M. Sun, W. Shi, J. Cen, J. Zhao and K. Xiao, Org. Biomol. Chem., 2018, 16, 6350-6357.

8 Q. Huang, Q. Li, Y. Chen, L. Tong, X. Lin, J. Zhu and Q. Tong, Sens. Actuators, B, 2018, 276, 82-88.

9 R. Wang, R. Wang, D. Ju, W. Lu, C. Jiang, X. Shan, Q. Chen and G. Sun, Analyst, 2018, 143, 5834-5840.

10 A. Chabok, M. Shamsipur, A. Yeganeh-Faal, F. Molaabasi, K. Molaei and M. Sarparast, Talanta, 2019, 194, 752-762.

11 M. H. Lee, J. S. Kim and J. L. Sessler, Chem. Soc. Rev., 2015, 44, 4185-4191.

12 P. Wu, X. Hou, J.-J. Xu and H.-Y. Chen, Nanoscale, 2016, 8, 8427-8442.

13 Q. Yang, J. Li, X. Wang, H. Peng, H. Xiong and L. Chen, Biosens. Bioelectron., 2018, 112, 54-71.
14 F. Q. Schafer and G. R. Buettner, Free Radicals Biol. Med., 2001, 30, 1191-1212.

15 R. A. Britten, J. A. Green and H. M. Warenius, Int. J. Radiat. Oncol., Biol., Phys., 1992, 24, 527-531.

16 M. S. Kasibhatla, S. D. Teeter and O. M. Colvin, Biomarkers, 2012, 17, 671-691.

17 M. H. Lee, J. L. Sessler and J. S. Kim, Acc. Chem. Res., 2015, 48, 2935-2946.

18 Y. Wang, L. Zhang, X. Zhang, X. Wei, Z. Tang and S. Zhou, ACS Appl. Mater. Interfaces, 2016, 8, 5833-5846.

19 J. Lai, B. P. Shah, E. Garfunkel and K. B. Lee, ACS Nano, 2013, 7, 2741-2750.

20 S. Santra, C. Kaittanis, O. J. Santiesteban and J. M. Perez, J. Am. Chem. Soc., 2011, 133, 16680-16688.

21 M. H. Lee, J. Y. Kim, J. H. Han, S. Bhuniya, J. L. Sessler, C. Kang and J. S. Kim, J. Am. Chem. Soc., 2012, 134, 1266812674.

22 X. Wu, X. Sun, Z. Guo, J. Tang, Y. Shen, T. D. James, H. Tian and W. Zhu, J. Am. Chem. Soc., 2014, 136, 3579-3588.

23 Y. Hu and F. Zeng, Mater. Sci. Eng. C, 2017, 72, 77-85.

24 Y. Liu, Q. Pei, L. Chen, Z. Li and Z. Xie, J. Mater. Chem. B, 2016, 4, 2332-2337.

25 S. Erbas-Cakmak, S. Kolemen, A. C. Sedgwick, T. Gunnlaugsson, T. D. James, J. Yoon and E. U. Akkaya, Chem. Soc. Rev., 2018, 47, 2228-2248.

26 H. Li and J. C. Vaughan, Chem. Rev., 2018, 118, 9412-9454.

27 K. Motyka, J. Hlaváč, M. Soural and P. Funk, Tetrahedron Lett., 2010, 51, 5060-5063.

28 K. Motyka, J. Hlaváč, M. Soural, P. Hradil, P. Krejčí, L. Kvapil and M. Weiss, Tetrahedron Lett., 2011, 52, 715-717.

29 A. K. Jain, M. G. Gund, D. C. Desai, N. Borhade, S. P. Senthilkumar, M. Dhiman, N. K. Mangu, S. V. Mali, N. P. Dubash, S. Halder and A. Satyam, Bioorg. Chem., 2013, 49, 40-48.

30 M. Soural, J. Hlaváč, P. Hradil, I. Fryšová, M. Hajdúch, V. Bertolasi and M. Maloň, Eur. J. Med. Chem., 2006, 41, 467-474.

31 V. Leen, T. Leemans, N. Boens and W. Dehaen, Eur. J. Org. Chem., 2011, 4386-4396.

32 J. Roy, T. X. Nguyen, A. K. Kanduluru, C. Venkatesh, W. Lv, P. V. N. Reddy, P. S. Low and M. Cushman, J. Med. Chem., 2015, 58, 3094-3103.

33 D. Giustarini, F. Galvagni, A. Tesei, A. Farolfi, M. Zanoni, S. Pignatta, A. Milzani, I. M. Marone, I. Dalle-Donne, R. Nassini and R. Rossi, Free Radicals Biol. Med., 2015, 89, 972-981. 